\title{
Management of giant cell arteritis among general practitioners from Genoa, Italy: a web-based survey
}

\author{
P. Clini' ${ }^{1}$, A. Stimamiglio², D. Camellino ${ }^{3}$, E. Hysa', M. Cutolo', M.A. Cimmino' \\ ${ }^{1}$ Research Laboratory and Academic Division of Clinical Rheumatology, Department of Internal Medicine, \\ University of Genova, Italy; 'General Practice, Azienda Sanitaria Locale 3 "Genovese", Genova, Italy; \\ ${ }^{3}$ Division of Rheumatology, "La Colletta" Hospital, Azienda Sanitaria Locale 3, Arenzano, Italy
}

\begin{abstract}
SUMMARY
Giant cell arteritis (GCA) is the most common form of vasculitis of the adult. General practitioners (GPs) are usually the first physicians who take care of GCA patients. In this study, the awareness of GPs from Genoa, Italy, regarding GCA was investigated by a web-based survey.

A web-based questionnaire was sent by mail to 775 Italian GPs. It included 12 multiple choice questions regarding practice seniority, practice population size, number of GCA patients followed, and GPs' diagnostic and therapeutic approach.

Of the 775 GPs involved, $76(9.8 \%)$ answered. Thirty-three/75 (44\%) declared that they did not see patients with GCA and the remaining $42(56 \%)$ that they diagnose between one and two patients per year. New headache onset was the presenting feature of GCA for the majority of GPs (78.3\%). GCA was diagnosed on the basis of clinical presentation alone by $35.2 \%$ of them, of temporal artery biopsy by $49.3 \%$, and by imaging, including ultrasound and magnetic resonance imaging, by $15.5 \%$. The referral pattern was mainly toward rheumatologists $(68.5 \%)$. Only $27.8 \%$ GPs declared they start treatment at the first clinical suspicion, with the others waiting for laboratory and imaging examinations or specialist consultation. The doses of glucocorticoids used were in keeping with current guidelines.

The management of GCA by GPs from Genoa is in general correct, with the exceptions of excessive confidence in headaches for diagnosis and of the timing of GC initiation. These points suggest that a program of information and education for GPs is warranted.
\end{abstract}

Key words: Temporal arteritis; giant cell arteritis; general practice; web-based survey.

Reumatismo, 2020; 72 (4): 207-212

\section{INTRODUCTION}

iant cell arteritis (GCA) is a large ves-

J sel vasculitis affecting elderly people, with the highest incidence among persons aged between 70 and 79 years (1). The frequency of this condition is probably higher than commonly believed (2) and is expected to increase, because of the increasing age of the general population. It has been estimated that between 2014 and 2050, more than 3 million people will have been diagnosed with GCA in Europe, North America and Oceania (3). General practitioners (GPs) are usually the first physicians who take care of patients presenting with complaints of GCA (4). Since early diagnosis and treatment is the key to reducing and possibly abolishing the feared complication of permanent sight loss (5), GPs should be able to correctly suspect, diagnose and manage this condition. In this study, awareness of GPs from the area of Genoa, Ligurian coast, Italy, regarding GCA was investigated by a web-based survey.

\section{MATERIALS AND METHODS}

A web-based questionnaire was sent by mail to 775 GPs working in the $3^{\text {rd }}$ Local
Corresponding author: Marco A. Cimmino Cattedra e Divisione di Reumatologia Dipartimento di Medicina Interna Università di Genova E-mail: cimmino@unige.it 
Health Unit of Genoa, a town located in North-Western Italy. Because no specific instrument exists to assess the management of GCA by GPs, the questions were developed on the basis of the current literature and personal experience. The questionnaire comprised 12 multiple choice questions regarding practice seniority, practice population size, number of incident GCA cases seen in the last years, number of GCA patients the GPs cared for, and their diagnostic and therapeutic approach. This computer-assisted web interview was run on the Google ${ }^{\circledR}$ platform. The questionnaire was sent for the first time on February 2, 2019 with a reminder on February 28.

Table I - Questions asked to the general practitioners.

\begin{tabular}{|l|}
\hline 1. In your practice, how many GCA patients do you see in average per year? \\
\hline$\square$ It's a condition I do not see in my practice \\
$\square$ 1-2 patients per year \\
$\square$ 3-4 patients per year \\
$\square$ More than 4 patients per year \\
\hline 2. In this moment, for how many patients with GCA do you care for? \\
\hline$\square$ 0-2 patients \\
$\square$ 3-10 patients \\
$\square$ 11-20 patients \\
$\square$ More than 20 patients \\
\hline 3. In your experience, which is the typical age range at GCA onset? \\
\hline$\square$ 40-50 years \\
$\square>51$ years \\
\hline 4. Which is the most frequent clinical manifestation of GCA in your patients? \\
\hline$\square$ Systemic signs (fever, weight loss, asthenia, ...) \\
$\square$ New-onset headache \\
$\square$ Arthralgia \\
$\square$ Visual impairment \\
\hline . Which
\end{tabular}

5. Which laboratory investigations do you request for confirmation of diagnosis in a patient with suspected GCA?

ESR/CRP

Blood cell count

IgM rheumatoid factor/anti cyclic citrullinated peptides antibodies

All of the above

6. To diagnose GCA, what do you use mainly?

$\square$ Clinical presentation

Temporal artery biopsy

Imaging

7. If you use imaging to confirm the diagnosis of GCA, which method do you prefer?

Ultrasound

Contrast-enhanced computed tomography (CT) or magnetic resonance imaging (MRI)

Positron emission tomography (PET)

I do not use imaging to confirm the diagnosis of GCA

8. If you suspect GCA, to which specialist do you refer your patient?

Rheumatologist

Immunologist

Internal medicine specialist

Ophthalmologist

Neurologist

None of them, I manage the patient by myself 
Continue $\gg>$

\begin{tabular}{|l|}
\hline 9. When do you start treatment in your patients with GCA? \\
$\square$ At first clinical suspicion \\
$\square$ After obtaining the results of the examinations requested \\
$\square$ After specialist consultation \\
$\square$ I do not start treatment by myself, but transfer it to the specialist consulted \\
\hline 10. Which treatment do you start in your patient with GCA? \\
$\square$ Prednisone (or equivalent) $>50$ mg/day \\
$\square$ Prednisone (or equivalent) $25-50$ mg/day \\
$\square$ Prednisone (or equivalent) $<25$ mg/day \\
$\square$ Other... \\
\hline 11. For how many subjects do you care for? \\
$\square$ 1-500 people \\
$\square$ 501-1000 people \\
$\square>1000$ people \\
\hline 12 . Which is your practice seniority? \\
$\square$ 0-10 years \\
$\square$ 11-20 years \\
$\square$ 21-30 years \\
$\square$ More than 30 years
\end{tabular}

It is shown in Table I. The Kruskall-Wallis test was used to analyze the answers to the questionnaire according to the characteristics of the GPs.

\section{RESULTS}

Of the 775 GPs involved, $76(9.8 \%)$ answered. The questionnaires with complete answers were $64(84.2 \%)$; in the remaining 12 , one or more answers were lacking. As a result, the percentage of answers was calculated on the total number of respondents for each question.

This group of GPs was relatively old with $80.8 \%$ reporting 20 years or more of practitioner seniority. Seventeen/72 (23.6\%) GPs had a practice population size of less than 500 people, $8(11.1 \%)$ between 500 and 1000 people, and $47(65.3 \%)$ more than 1,000 people.

Thirty-three/75 (44\%) GPs answered that they did not see patients with GCA and the remaining $42(56 \%)$ that they diagnose between one and two patients per year. GPs who said they do not see patients with GCA were asked to answer the remaining questionnaire anyway. The prevalence of patients affected by GCA among the subjects cared for by GPs was between 0 and 2 for $93.3 \%$ of them, and between 3 and 10 for $6.7 \% .90 \%$ of GPs declared that the age of onset of GCA in their patients was more than 50 years, whereas it was between 40 and 50 years in the remaining $10 \%$.

New headache onset was the presenting feature of GCA for 54/69 (78.3\%) GPs, systemic inflammatory symptoms for 8 (11.1\%), arthralgia for $4(5.8 \%)$, and visual problems for 3 (4.3\%). Of these GPs, 25/71 (35.2\%) diagnosed GCA on the basis of clinical presentation alone, $35(49.3 \%)$ requesting also temporal artery biopsy and $11(15.5 \%)$ asking for imaging. As far as imaging is concerned, temporal artery ultrasound (US) was considered by 16 GPs, magnetic resonance imaging (MRI)/computed tomography (CT) by 15 , and positron emission tomography (PET) associated with US by one. One GP performed diagnostic US by himself. Twenty-nine GPs $(47.5 \%)$ confirmed they do not use imaging. The laboratory investigations requested to patients with suspected GCA were erythrocyte sedimentation rate (ESR) and/or C-reactive protein (CRP) for 24/72 $(33.3 \%)$ GPs, and the combination of all investigations proposed in the questionnaire (blood cell count, ESR, CRP, IgM rheumatoid factor, and anti-citrullinated protein antibodies) for the remaining $48(66.7 \%)$. Five $(6.8 \%)$ GPs answered they do not 
refer their patients to any specialist and follow them by themselves. The rheumatologist was the specialist to whom 50/73 $(68.5 \%)$ GPs referred their patients; immunologist $(3,4.1 \%)$, internal medicine specialist $(5,6.8 \%)$, and neurologist $(9$, $12.3 \%$ ) followed. The ophthalmologist was cited by 3 GPs only, but always in conjunction with another specialist among those listed above.

Only 20/72 (27.8\%) GPs declared they start treatment at the first clinical suspicion, $24(33.3 \%)$ after they obtain the results of laboratory and imaging examinations, 15 $(20.8 \%)$ after the results of specialist consultation, and $13(8.1 \%)$ answered they do not initiate treatment but transfer it to the reference specialist. Twenty-seven/69 $(39.1 \%)$ GPs started treatment with dosages of prednisone higher than $50 \mathrm{mg} /$ day, $40(58 \%)$ with dosages between $25 \mathrm{mg} /$ day and $50 \mathrm{mg} / \mathrm{day}$, and none of the GPs used less than $25 \mathrm{mg}$ prednisone daily. One GP specified using $1 \mathrm{mg}$ prednisone per $\mathrm{kg}$ body weight and another associated prednisone with both NSAIDs and low dose aspirin.

When the answers to the questionnaire were analyzed according to the duration of the GPs' professional activity and the number of subjects they cared for, no differences were found.

\section{DISCUSSION AND CONCLUSIONS}

To our knowledge, this is the first study on the behavior of Italian GPs toward GCA and its management. Unfortunately, the percentage of respondents $(9.8 \%)$ was low and does not permit us to generalize the results. However, this is a common finding in surveys of GPs. In another study on GCA in the UK general practice, the response rate to a questionnaire was $25 \%$ (6). In the same group of GPs from Genoa we interviewed, the response rate for previously administered questionnaires was $2.4 \%$ (renal insufficiency), $12.3 \%$ (alcoholism), and $15.6 \%$ ( $\beta$-blockers in cardiac insufficiency). Therefore, the percentage of responders was similar across countries and different topics. Among the possible reasons for this scarce compliance, the increasing number of web-based surveys the GPs receive, their lack of time because of the many bureaucratic duties, and the modest interest in a rare condition such as GCA can be hypothesized.

GPs who said they are used to seeing patients with GCA affirmed that they see one or two patients per year. This is in keeping with the figure of another study (7) on British full-time GPs. GPs emphasized headache as main symptom at presentation. Although this may be considered correct, the observation suggests that, in general practice, the cranial form of GCA is more frequently recognized, whereas the noncranial one may be underdiagnosed. GPs appeared to over-rely on headache for the diagnosis of GCA also in the study performed in the UK (6). In view of the fact that half of GCA patients do not complain of the typical headache (8), this may result in a significant loss of correct diagnoses. The laboratory and imaging approach to GCA diagnosis was correct and in accordance with the recently published guidelines by the British Society for Rheumatology (BSR) (9). It strongly recommends that all patients with GCA should have at least one confirmatory diagnostic test, which could be either temporal artery biopsy or temporal and axillary artery ultrasound. This approach, described by nearly $65 \%$ of respondents, was mainly based on temporal artery biopsy, as expected. This is reasonable, since it is among the timehonored American College of Rheumatology criteria for the classification of GCA (10). US was often cited as diagnostic aid by Italian GPs whereas it was not cited at all by their British counterparts (6). This may be due to the fact that the British study was performed 2 years earlier and further evidence has been accumulating in the meantime on the proficiency of temporal artery US (11). Moreover, US was not acknowledged in the previous BSR guidelines (12). From our data, it is impossible to understand if MRI, which was cited by $22.3 \%$, was used to diagnose GCA according to the relevant guidelines (11) or 
to exclude neurological differential diagnoses. The second hypothesis is more likely, due also to the limited access to $3 \mathrm{~T}$ machines in our area; high-field MRI machines are usually employed for this type of imaging (11). Referral to rheumatologists, who were cited as reference specialists by $68.5 \%$ of GPs, was frequent whereas referral to ophthalmologists was only cited by three of them, who also indicated visual problems as presenting features of their patients. In the UK, GPs referred their patients to rheumatologists in $38.3 \%$ of occasions (6), which figure is definitely lower than in Genoa.

All GPs interviewed used prednisone starting dosages higher than $25 \mathrm{mg} /$ day and $39 \%$ higher than $50 \mathrm{mg} / \mathrm{day}$; the recommended dosage is 40-60 mg/day (9) but, due to the design of the question, it was impossible to ascertain exactly how many GPs were following this guideline. Nonetheless, it seems that in general the starting dosage of prednisone was correct. On the contrary, the question on the time of onset of treatment yielded less satisfactory results. The BSR guidelines indicate that patients in whom GCA is strongly suspected should be immediately treated with highdose glucocorticoids (GC). Contrary to this statement, only $27.8 \%$ of GPs started GC at initial clinical suspicion. The remaining waited for the results of biopsy, laboratory and imaging examinations, and specialist consultation, or prescribed treatment by a specialist. This figure is comparable to the $35.6 \%$ of British GPs who would not initiate treatment prior to referral (6). In Italy, this approach would generally imply a long time interval between the initial suspicion of GCA and initiation of treatment, which fact is associated with an unacceptable high risk of visual loss (5). For this reason, fasttrack referral pathways have been set up in several countries, where initial diagnostic evaluation and treatment of patients with suspected GCA is performed within $24 \mathrm{~h}$ of referral (13). This pathway, which has been associated with a reduction in reported rates of GCA-related sight loss compared with conventional care, has not been implemented in our area to date. Therefore, GPs should be encouraged to start treatment as soon as they conceive a strong clinical suspicion of GCA, even if they have not obtained confirmation, because the risk of toxicity caused by GC treatment is acceptably low in case of misdiagnosis.

In conclusion, we have shown that the management of GCA by Ligurian GPs is in general correct, with the exceptions of an excessive confidence in headaches for diagnosis and of the timing of GC initiation. These points suggest that a program of information and education for GPs is warranted.

\section{Acknowledgements}

We are grateful to all the general practitioners who responded to the questionnaire for their participation. Prof. Carlo Pesce, Associate Professor of Pathology, kindly reviewed the manuscript.

\section{Conflict of interest}

The authors declare no potential conflict of interests. This research did not receive any specific grant from funding agencies in the public, commercial, or not-for-profit sectors.

\section{REFERENCES}

1. Salvarani C, Cantini F, Boiardi L, Hunder GG. Polymyalgia rheumatica and giant-cell arteritis. N Engl J Med. 2002; 347: 261-71.

2. Cimmino MA, Camellino D. Large vessel vasculitis: is it more common than usually assumed? Reumatismo. 2017; 69: 143-6.

3. De Smit E, Palmer AJ, Hewitt AW. Projected worldwide disease burden from giant cell arteritis by 2050. J Rheumatol. 2015; 42: 119-25.

4. Caylor TL, Perkins A. Recognition and management of polymyalgia rheumatica and giant cell arteritis. Am Fam Physician. 2013; 88: 676-84.

5. Ezeonyeji AN, Borg FA, Dasgupta B. Delays in recognition and management of giant cell arteritis: results from a retrospective audit. Clin Rheumatol. 2011; 30: 259-62.

6. Helliwell T, Muller S, Hider SL, et al. Challenges of diagnosis and management of giant cell arteritis in general practice: A multimethods study. BMJ Open. 2018; 8: 1-8.

7. Barraclough K, Mallen CD, Helliwell T, et al. Diagnosis and management of giant cell arteritis. Br J Gen Pract. 2012; 62: 329-30. 
8. Smetana GW, Shmerling RH. Does this patient have temporal arteritis? JAMA. 2002; 287: 92-101.

9. Mackie SL, Dejaco C, Appenzeller S, et al. British Society for Rheumatology guideline on diagnosis and treatment on giant cell arteritis. Rheumatology (Oxford). 2020; 59: e1-e23.

10. Hunder GG, Bloch DA, Michel BA, et al. The American College of Rheumatology 1990 criteria for the classification of giant cell arteritis. Arthritis Rheum. 1990; 33: 1122-8.

11. Dejaco C, Ramiro S, Duftner C, et al. EULAR recommendations for the use of imaging in large vessel vasculitis in clinical practice. Ann Rheum Dis. 2018; 77: 636-43.

12. Dasgupta B, Borg FA, Hassan N, et al. BSR and BHPR guidelines for the management of giant cell arteritis. Rheumatology (Oxford). 2010; 49: 1594-7.

13. Patil P, Williams M, Maw WW, et al. Fast track pathway reduces sight loss in giant cell arteritis: results of a longitudinal observational cohort study. Clin Exp Rheumatol. 2015; 33: S103-6. 